\title{
A Comparative Assessment of an Indoor Localization Framework for Building Emergency Response
}

\author{
Nan $\mathrm{Li}^{{ }^{1 *}}$, Burcin Becerik-Gerber ${ }^{2}$, Lucio Soibelman ${ }^{2}$, Bhaskar Krishnamachari ${ }^{3}$ \\ ${ }^{1 *}$ Department of Construction Management, Tsinghua University, Beijing 100084, China (corresponding \\ author, tel: (+86)10-62796834, email: nanli@tsinghua.edu.cn) \\ ${ }^{2}$ Sonny Astani Department of Civil and Environmental Engineering, University of Southern California, \\ Los Angeles, CA 90089, United States \\ ${ }^{3}$ Ming Hsieh Department of Electrical Engineering, University of Southern California, Los Angeles, CA \\ 90089, United States
}

\begin{abstract}
Building emergencies, especially structure fires, are threats to the safety of both building occupants and first responders. It is difficult and dangerous for first responders to perform search and rescue in an unfamiliar environment, sometimes leading to secondary casualties. One way to reduce such hazards is to provide first responders with timely access to accurate location information. To address this challenge, the authors have developed a radio frequency based indoor localization framework, for which novel algorithms were designed for two different situations: one where an existing sensing infrastructure exists in buildings and one where an adhoc sensing infrastructure must be deployed. This paper presents a comparative assessment of this framework under different situations and emergency scenarios, and between simulations and field tests. The paper first presents an assessment of the framework in field tests, showing that it achieves room-level accuracies above $82.8 \%$ and $84.6 \%$ and coordinate-level accuracies above $2.29 \mathrm{~m}$ and $2.07 \mathrm{~m}$, under the two situations, respectively. Moreover, the framework demonstrates considerable robustness in the tests, retaining a room-level accuracy of $70 \%$ or higher when the majority of sensing infrastructure is damaged. This paper then synthesizes results from both simulations and field tests, and demonstrates how the framework can be adapted to different situations and scenarios while consistently yielding satisfactory localization performance.
\end{abstract}

Keywords: Assessment; Indoor localization; Emergency response; Radio frequency; Simulation; Field test 


\section{Background}

Building emergencies especially structure fires are big threats to the safety of building occupants and first responders. For example, public fire departments across the U.S. attended 487,500 fires in buildings in 2013, which caused 2,755 deaths and 12,200 injuries [1]. When emergencies occur, unfamiliar environments are difficult and dangerous for first responders to search and rescue, sometimes leading to secondary casualties. Statistics show that $87 \%$ of fire-related firefighter fatalities and injuries occur in structure fires [2]. A total of 159 firefighters died between 20002011 in the U.S. when responding to structure fires, one major cause of which was firefighters getting lost $[3,4]$. One way to reduce such hazards is to provide firefighters with timely access to accurate location information. Their increased awareness of own locations within the spatial context would significantly reduce the possibility of getting lost in buildings as well as the associated fatalities and injuries.

It is also of critical importance for an incident commander to know the locations of first responders in real time, so that decision-making processes are made faster and more informed. When an emergency happens, first response teams are sent to carry out search and rescue operations. In most cases, searching for occupants is a manual process and requires a complete inspection of all indoor spaces. Such blind search process is highly inefficient and could be prohibited by fire, smoke or structural damages. Reducing the time spent on searching for occupants has great potential to reduce chances of fatalities and injuries of trapped occupants, and it can be achieved by making the locations of trapped occupants more transparent to first responders at emergency scenes.

Access to location information during emergency response operations is far from being automated and efficient. Currently, after a size-up of an emergency, which evaluates the severity of an incident and estimates required resources based on visual inspections from outside a building, first response teams are sent in to the building, usually in groups of four, to perform various tasks such as fire attack, ventilation, and search and rescue. The deployed first responders communicate over radios with an incident commander outside the building, who marks tasks and locations of the deployed teams in a command post and updates this information based on vocal reports received from the deployed teams. However, it is challenging to keep this information organized and updated, considering the ever-changing situations inside a building, especially when multiple teams use multiple radio channels to communicate. Access to real-time location information, if made possible, would enable an incident commander to better monitor and guide 
the deployed first responders. This would lead to reduction of their chances of getting lost or trapped, and improvement of their efficiency in performing assigned tasks. On the other hand, search for trapped occupants is usually done in two rounds. During a primary search, first responders traverse the building, determine a rough number and location of trapped building occupants and rescue them. During a secondary search, first responders make sure all spaces are thoroughly searched, and rescue occupants who are still trapped. Although radios (and in some cases thermal cameras) are used to help detect the occupants at emergency scenes, the search process is generally low-tech and blind. First responders usually have little clue of how many occupants are trapped, where they are, and how to reach them. There is a need for an indoor localization solution that enables first responders to obtain real-time location estimations of both themselves and trapped occupants during emergencies, so that they can prioritize spaces that are more likely to have occupants when planning the search and rescue routes, and increase their own safety during emergency response operations.

\section{A Radio Frequency Based Indoor Localization Framework}

\subsection{Need Analysis}

Given the significance of indoor location information at building emergency scenes, the authors have carried out the following research efforts that have led to the development of a novel indoor localization framework using radio frequency $(\mathrm{RF})$ technologies.

First, the authors examined the relative importance of indoor location information among a list of nineteen information items that may be useful during building emergencies [5]. Interactive interviews were designed and conducted with first responders from the Los Angeles Fire Department (LAFD). During the interactive interviews, imaginary building emergencies were presented to the interviewees who were asked to command an emergency operation with the help of the provided information items. Based on the interview results, for each information item, its importance was examined from three perspectives including the order it was requested during the interviews, the frequency it was updated, and its overall value ranked by the interviewees. The importance of each information item was assessed in a quantitative manner, and it was found that indoor location information was one of the most important information items in all stages of building emergency response operations.

A survey was also carried out among first responders across the U.S. to examine the requirements for indoor localization at building emergency scenes [5]. The survey was motivated by the fact 
that, with the rapid adoption of various sensing tools, such as remote sensing [6-8], geographic information systems (GIS) [9-11], thermal imaging cameras [12], and mobile computing and communication devices $[13,14]$, that are used in the emergency response practices, there is need for an effective tool that can aid the search for victims and tracking of deployed first responders. Most existing indoor localization solutions for building emergency response operations, either proposed in the academia [15-23] or are available in the market [24,25], were developed in the absence of clear knowledge about the needs of first responders if an indoor localization solution existed. This problem is even more obvious with indoor localization solutions developed for general purposes using various technologies such as inertial navigation systems (INS) [26-28], assisted GPS (AGPS) [29-31], and infrared [32-34], as well as a couple of RF technologies including ultra wide band (UWB) [35-37], radio frequency identification (RFID) [38-41], wireless local area network (WLAN) [42-45], and wireless sensor networks (WSN) [46-48]. For example, most existing solutions predominantly emphasized their high accuracies; however, none of them argued what level of accuracy is sufficient to support emergency response operations while not becoming over demanding in terms of supporting infrastructure or prior data input, or to what extent an accuracy should be retained when a solution is challenged by hazards such as fire and structural collapses. In this survey, the respondents were asked to select the top five important requirements from a list of eleven requirements. Based on the survey results, the following requirements were identified as critical when designing or evaluating indoor localization solutions for building emergency response operations: accuracy (focus on roomlevel), ease of on-scene deployment (up to $2.25 \mathrm{~min}$ for ad-hoc network setup), robustness (against physical damages), computational speed (approximately $40 \mathrm{~s}$ for location computation), and finally size and weight of devices (up to $107 \mathrm{~cm}^{3}$ and $1.15 \mathrm{~kg}$ ).

\subsection{Methodology}

Based on the above requirements, the authors designed two localization algorithms, named the

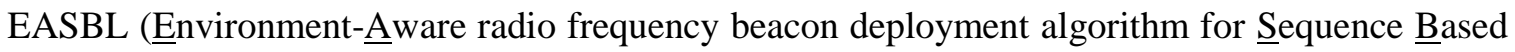
Localization) and the IMLE (Iterative Maximum Likelihood Estimation). Built on a sequence based localization schema [49], the EASBL was designed for situations, where ad-hoc sensor networks are needed at emergency scenes in the absence of existing sensing infrastructure. The algorithm has a dual-objective function that balances between localization accuracy and the deployment effort of ad-hoc networks. The likelihood of correct room-level location estimation is measured by location space quality, a metric that can be calculated based on geometries of the space and the deployment plan of devices. The deployment effort is measured by the number of 
devices to deploy, and the accessibility of locations to deploy the devices. The EASBL uses a Tabu search metaheuristic to improve the efficiency of searching for optimal plans for space division and device placement. The algorithm design and details as well as the mathematical formulation can be found in [50].

The IMLE was designed for situations, where existing sensing infrastructure can be accessed to collect RF data needed for location computation. Existing sensing infrastructure can be a network of any type of RF transmitters and transceivers installed in buildings for certain purposes, such as communications and security. This network of RF devices can be used for localization purposes during emergencies, provided that the information of its configuration, particularly the layout and specifications of the devices, is known. The IMLE integrates a maximum likelihood estimation (MLE) method for estimating the parameter values of a RF signal propagation model. Such adhoc estimation reflects the impacts of environmental factors on RF signal propagation. The model is then used to infer target locations from collected RF signals, which also adopts the MLE method. In addition, the IMLE introduces a novel iterative computational process, which integrates the spatial layouts and searches for room-level estimations that can lead to convergence after certain iterations or, if no converged estimations exist, estimations that are most likely to converge. This iterative process offsets the impact of wall-related RF signal attenuations on the localization accuracy. The details of the IMLE algorithm can be found in [51]. Both algorithms were evaluated extensively however, only under simulated building emergency scenarios, and reported promising results that demonstrated the potential of the algorithms in satisfying the aforementioned five requirements. It needs to be emphasized that both algorithms closely integrate building information modeling (BIM) as a source of indoor geometric information used in location computation, and a platform for user interaction and visualization of localization results.

As a continuous research effort, this paper further assesses the localization framework with two algorithms, in hopes of achieving the following objectives:

(1) Assess the performance of these two algorithms in field tests. Simulation based evaluation is advantageous in that the tests are easily repeatable, and that evaluations are carried out in a controlled environment so that the impact of a particular factor can be isolated and analyzed. However, simulation based evaluation is also challenged by the fact that the real-world environments are more complex and unpredictable, which may impact the performance of the indoor localization framework in ways that are unobservable in a simulation. Field tests are 
therefore necessary in order to examine the achievable performance of the algorithms under various constraints imposed by real-world environments. In this paper, the two aforementioned algorithms are examined in field tests conducted in a typical office building. A smartphone-based prototype was developed for the tests, and emergency scenarios parallel to those used in simulations were used for field evaluations.

(2) Evaluate the performance of the localization framework under different situations and scenarios. The EASBL and the IMLE were examined separately under situations they were respectively designed for. For either algorithm, the simulation based assessments and the field test based assessments were carried out in two consecutive stages. The simulations and field tests shared the same test bed, and emergency scenarios and situations, which provided the basis for comparisons. By providing a comparative evaluation of both algorithms, this paper synthesizes previous results, and demonstrates how the framework can be adapted to different situations and scenarios while consistently yielding satisfactory localization performance.

\section{Field Test Setup}

This section presents the design of the smartphone-based prototype used in the field tests, and explains the details of emergency scenarios and situations, based on which the field tests were conducted.

\subsection{Prototype Development}

The prototype consisted of three major components: RF transmitters, smartphones, and a localization server. The RF transmitters were off-the-shelf programmable routers (Figure 1a) [52]. Each transmitter, with a size of $113 \mathrm{~mm} \times 138 \mathrm{~mm}$ x $29 \mathrm{~mm}$ and a weight of 230 grams, contained a $400 \mathrm{MHz}$ processor, $32 \mathrm{MB}$ onboard memory, two built-in high power antennae, five Ethernet ports, and light-emitting diodes (LED) indicator lights. With a transmit power of 30 $\mathrm{dBm}$, the transmitter could create a $802.11 \mathrm{~b} / \mathrm{g} / \mathrm{n}$ wireless access point that was detectable up to fifty meters away in an open space. During the field test, in scenarios where an ad-hoc network was needed, the transmitters were used as beacon nodes in the network, and they were deployed by first responders at the beginning of the field test following the deployment plans developed by the EASBL algorithm. In scenarios, where existing sensing infrastructure was available, the transmitters were preinstalled in the test bed before the field tests began, and the information, including their mac addresses, service set identifiers (SSID), and locations, was recorded beforehand and made available for location computation. In either case, each transmitter had its 
own unique mac address and SSID, which were visible to any device that received the RF signal the transmitter broadcasted.

Figure 1: Transmitter (a) and smartphone (b) used in the prototype

The smartphones used in the prototype were also off-the-shelf smartphones (Figure 1b) [53] with a size of $116 \mathrm{~mm}$ x $60 \mathrm{~mm}$ x $14 \mathrm{~mm}$ and a weight of $169 \mathrm{~g}$. Each smartphone had a $600 \mathrm{MHz}$ processor, $256 \mathrm{MB}$ memory, extendable storage capacity, and built-in cellular, WiFi and Bluetooth modules, and relied on a built-in battery for power. It supported customized and thirdparty applications. A localization application was developed for this prototype (Figure 2) and installed in the smartphones. The application, when run by the user, would turn on the WiFi sensor in the smartphone, scan all detectable WiFi access points in the environment every five seconds, collect RF signal data, and forward it to a localization server, whose IP address was programmed into the application beforehand but could also be overwritten by user input. The data sent by the application to the server included the mac address, SSID and RSSI of every detected WiFi access point, the device ID of the phone, and a timestamp. The data transition could rely on either the WiFi or the cellular connection. The application also had a user interface that allowed users to monitor the data collection and transition processes, and to stop or resume the localization function whenever needed. In addition, an off-the-shelf time synchronization application was also installed in the smartphone. This application kept the clocks synchronized among all smartphones and the localization server during the field test. A few smartphones were pre-deployed and used as transceivers in the field tests.

Figure 2: Interface of the localization application

A remote localization server was set up as part of the prototype. Accessible to the incident commander, the localization server consisted of four major components: a webserver, an SQL database, a BIM platform, and a location computation module. The webserver was responsible for receiving the signal data that were sent by the smartphone application in a JSON format, parsing the data, and passing the data to the SQL database. The SQL database, upon the receipt of the data, checked the integrity of the data, and stored it in two separate tables, one for real-time processing, and the other for data backup. The SQL database was also used for maintaining additional data required for location computation. This data included the location, mac addresses, SSID and transmit power of existing transmitters and transceivers, the mapping between IDs of 
the phone users and the phone device IDs. The SQL database also included a table that kept a record of all location computation results. This table not only allowed further analysis of the localization results, but also had the potential to be used for supporting an extended target tracking function. The BIM platform (Figure 3), based on a commercial BIM authoring tool [54] and a customized add-on development, was used to interact with the BIM model of the test bed building that had emergency situations. The BIM platform extracted building geometric information from the BIM models, and used it for interpreting the layout of existing sensing infrastructure or the ad-hoc networks, as well as supporting location computation. It also provided a GUI that allowed users to define the sensing area, indicate the availability of existing sensing infrastructure, monitor location computation progress, and see visualized localization results. Lastly, the location computation module was responsible for processing the data retrieved from the SQL database and the BIM models, estimating targets' locations by implementing the EASBL and IMLE algorithms, and sending the location computation results back to the SQL database for record and to the BIM platform for visualization. Figure 4 shows the connection of the above components of the prototype and the data flow in the field tests.

Figure 3: Snapshot of the BIM platform

Figure 4: Data flow in the field tests

\subsection{Field Test Scenarios, Procedures and Test Bed Setup}

The field tests were carried out in a representative floor of a typical office building on the University of Southern California campus. This floor has an area of about $1800 \mathrm{~m}^{2}$, containing a total of 14 single occupancy offices, and 12 multi occupancy conference rooms or labs. The hallway has a length of about $100 \mathrm{~m}$ comprised of four linear segments. Each linear segment was treated as a separate room in the tests. Instances when test subjects were in the hallway were included when calculating the room-level accuracy. Two imaginary building fire emergency scenarios were used in the field tests (Figure 5). Both scenarios were designed based on the suggestions from a number of first responders, and were reviewed and verified by two incident commanders from the LAFD in terms of the representativeness of the incidents and the scope of the sensing areas. In scenario 1 (Figure 5, a, b), two single offices were on fire (marked as rooms on fire in the figure). Occupants in these offices, all neighboring offices, and offices and conference room that were across the hallway and had doors open to the hallway (i.e., all rooms within the sensing area boundary) had to be evacuated. Due to the spreading smoke, visibility in 
the hallway outside the offices was low, resulting in an increased risk to the first responders. The sensing area was defined by the sensing area boundary in Figure $5(\mathrm{a}, \mathrm{b})$ with a size of $221 \mathrm{~m}^{2}$. In scenario 2 (Figure 5, c, d), fire started in a lab and soon spread to another lab across the hallway (marked as rooms on fire in the figure). All labs on the east side of the floor were shut down for fire attack and search $\&$ rescue (i.e., all rooms within the sensing area boundary). Visibility in the hallway was low due to the smoke. The sensing area was defined by the sensing area boundary in Figure $5(\mathrm{c}, \mathrm{d})$ with a size of $729 \mathrm{~m}^{2}$.

In both emergency scenarios, two situations were tested, including situation 1 (Figure 5, a, c), where no existing sensing infrastructure existed in the building, an ad-hoc sensing network was required, and the EASBL algorithm was used; and situation 2 (Figure 5, b, d), where existing sensing infrastructure was available, and the IMLE algorithm was used. The layouts of the devices in both scenarios and both situations are illustrated in Figure 5. For each combination of scenario and situation, a field test was designed and repeated twice. A total of eight field tests were conducted.

Figure 5: Simulation scenarios and device layouts

Based on the common resource dispatch rules used by fire departments in the U.S., the following resources would be dispatched upon the receipt of an emergency call from the test bed building: four engine companies, two truck companies, two rescue ambulances, one battalion chief, and one emergency medical service captain. In both scenarios, based on the discussions with incident commanders from the LAFD, the following assumptions about resource allocation and actions taken by first responders and trapped occupants within the sensing area were made: the truck companies, ambulances, battalion chief and medial captain would be working outside the building. Three engine companies would be deployed to work inside the building, with a fourth engine company standing by. Each engine company consisted of four first responders. Two deployed engine companies would be assigned to attack the fire, with three first responders in each company working together within the sensing area, and one first responder working outside the area on water supplies. The teams would first lay out hoses, and deploy an ad-hoc sensor network, if needed. They would then work in or around burning rooms until the fire was put out. One deployed engine company would be assigned to search and rescue trapped occupants. The team would traverse all rooms in the sensing are and, when trapped occupants were found, escort them to a safe zone. In both scenarios, it was assumed that five occupants were trapped in four rooms when the first response teams arrived. Two of them moved around, trying to find their way out 
but blocked by fire and smoke, until they were rescued by the first responders. Three of them stayed in their rooms and wait for the rescue. The emergency situation was assumed to be under control within 20 minutes after the first response teams were deployed. To mimic this process, each field test was designed to take a maximum of 20 minutes. A total of sixteen test subjects participated in the field tests. One subject assumed the role of a battalion chief, who operated the localization solution and commanded the emergency response operation from outside the sensing area. Among the rest of the fifteen subjects, ten assumed the role of first responders in three engine companies, and five assumed the role of trapped occupants. These fifteen subjects acted as targets in the field tests, and they were always monitored and located when they were inside the sensing area during the field tests. Every target was equipped with a smartphone that was synchronized with the server. The localization application was installed in the smartphones. Every target was also given a stopwatch, and a unique script that listed a number of locations the target needed to traverse. A target was instructed to take an action every 15 seconds paced by the stopwatch. An action can be either moving to the next location indicated in the script, or staying at the current location. A target needed to perform a total of 60 actions in every field test, including actions taken by a group of first responders in situation 1 to deploy ad-hoc networks. There were 66 different locations that a target might be instructed to visit. Scattered in the sensing area, these locations were marked with numbered sticky notes for easy recognition, and their locations were measured beforehand and used as the ground truth. All subjects went through onehour long training before the field tests. During the training, they were explained the purpose and procedure of the field test, their respective roles, and the instructions in their individual scripts. They were also instructed about how to use the two applications installed in their smartphones. In addition, the subjects were walked through the test bed during the training to get familiar with the locations of the sticky notes, so that they could quickly navigate in the building during the field tests.

\section{Field Test Results}

This section presents the results reported from eight field tests conducted in the test bed building.

\subsection{Evaluation of the Framework with No Existing Sensing Infrastructure}

\subsubsection{Localization Accuracy and Deployment Effort}

For situation 1, where there is no existing sensing infrastructure at emergency scenes, the deployment of an ad-hoc sensor network was needed, and the EASBL algorithm was used for 
location computation. In the field tests, an optimal Tabu size of 10 as reported in the simulation was used in the EASBL. For each scenario, the average accuracies of the two repeated field tests are presented in Table 1. The localization accuracy with a proximity schema, coupled with optimal transmitter placements reported by the EASBL, was also calculated. The proximity schema assumed the location of the nearest transmitter to a target as the target's location [41]. The proximity schema is one of the most widely used localization schema. It usually outperforms range-based schema and does not require prior data input as fingerprinting schema does, and is applicable to data collected by the ad-hoc networks. The results are presented in Table 1.

Table 1: Localization accuracy of the EASBL algorithm and the optimal placement with proximity schema in the field tests

In all of the four field tests in situation 1, the room level accuracy achieved by the EASBL algorithm was above $80 \%$ and the coordinate-level accuracy was above $2.5 \mathrm{~m}$. Specifically, the respective average room-level accuracies in scenario 1 and scenario 2 were $82.8 \%$ and $83.7 \%$, and the respective average coordinate-level accuracies in scenario 1 and scenario 2 were $2.12 \mathrm{~m}$ and $2.29 \mathrm{~m}$. These results were better than those with the proximity schema, suggesting the competence of the EASBL. It was observed that the room-level accuracy of the EASBL was significantly better but the difference between the coordinate-level accuracies was not that significant. This phenomenon was a reflection of the fact that the EASBL was particularly designed to improve room-level localization accuracy, by strategically selecting the placement of RF devices, whereas the coordinate-level accuracy did not necessarily benefit from the optimized layout. Moreover, further analysis revealed that $41.7 \%$ of incorrect room-level estimations by the EASBL were within rooms neighboring the correct rooms. This finding suggests that even an incorrect estimation could potentially be used to indicate in which multi-room zone a target may be located. This is important, especially when first responders fail to find trapped occupants in estimated rooms in the first attempt and need clues for secondary places to search. In general, the accuracies reported in the field test provided convincing evidence about the capability of the EASBL algorithm in providing reliable location information at emergency scenes.

\subsubsection{Robustness against Partial Loss of Deployed Transmitters}

Further analysis of the test data was carried out to evaluate the robustness of the EASBL against occurrence of loss of deployed transmitter. The ad-hoc networks deployed in the test bed included 11 and 13 transmitters in scenario 1 and scenario 2, respectively. For each scenario, beginning 
with the initial deployment, randomly selected transmitters were taken out, one at a time, and the accuracy was re-calculated only based on the data collected from the remaining transmitters. In order to offset the impact of randomness in selecting the transmitter to be taken out, for a specific number of remaining transmitters, the process was repeated 100 times. The minimum number of deployed transmitters to make sensing space division possible in the EASBL algorithm was 2 transmitters. The results are shown in Figure 6.

Figure 6: Assessment of robustness against partial loss of deployed transmitters

In scenario 1, when no more than 4 transmitters, or $36.4 \%$ of all transmitters, were removed, the room-level accuracy remained above $70 \%$ and the coordinate-level accuracy remained above 2.5 m. In scenario 2, when no more than 7 transmitters, or $46.2 \%$ of all transmitters, were removed, the room-level accuracy remained above $70 \%$ and the coordinate-level accuracy remained above $3.0 \mathrm{~m}$. Such limited impact of device losses on the localization accuracy provided a demonstration of the robustness of the EASBL algorithm under situation 1 in the field tests. However, when the device losses continued to increase, the impact became more obvious. A significant decline of accuracy was observed when 6 or more transmitters were removed in scenario 1, resulting in a room-level accuracy of below $60 \%$ and a coordinate-level accuracy of below $3.0 \mathrm{~m}$, and when 8 or more transmitters were removed in scenario 2, resulting in a roomlevel accuracy of below $60 \%$ and a coordinate-level accuracy of below $3.5 \mathrm{~m}$.

\subsubsection{Ease of On-Scene Deployment, Computational Speed, and the Size and Weight of Devices}

In addition to the accuracy and robustness, the aforementioned survey results also revealed that a localization solution should have ease of on-scene deployment, have fast computational speed, and use devices with desirable size and weight. The ease of on-scene deployment, measured by the deployment effort of ad-hoc networks, is improved by the inherent design of the EASBL algorithm. During the field tests, the ten subjects assuming the role of first responders were able to identify the specified locations for node deployment and set up an entire ad-hoc network within 90 seconds after the instructions were provided. While admittedly more time would be required for the ad-hoc network deployment at real-world emergency scenes due to conditions such as fire and smoke, the results from the test deployment were promising. Well trained first responders are likely to be able to complete the deployment within $135 \mathrm{~s}$, which is the maximum amount of time allowed to be spent on a deployment, as reported in the survey [5]. 
In terms of the computational speed, it was observed that one update of the location estimations, which involved collecting the data from the ad-hoc network, computing the targets' locations, and presenting the localization results in the BIM platform, took less than $5 \mathrm{~s}$, far less than $40.34 \mathrm{~s}$, which was reported as an appropriate amount of computational time in the survey [5]. Moreover, the Tabu search was used by the EASBL algorithm to quickly search for satisfactory solutions to initial ad-hoc networks deployment. The integration of this soft computing technique significantly improved the computational efficiency. However, the EASBL algorithm, when implemented in its current shape, required excessive computational time in computation of the transmitter deployment plan due to the delay in processing of building geometries, which was performed by the BIM authoring tool. The BIM tool was selected solely based on its availability regardless of its efficiency, as the scope of the research presented in this paper does not focus on improving the computational efficiency of processing building geometries. It was observed that the building geometry processing was responsible for over $90 \%$ of the total computational time. Since the processing of geometries occurs only when finding satisfactory solutions to transmitter deployment, the impact of low efficiency in geometry process was limited to the impediment of fast network deployment at the beginning of the emergency response operation.

Lastly, the sizes of the transmitters $\left(0.45 \mathrm{~cm}^{3}\right)$ and the smartphones/transceivers $\left(0.10 \mathrm{~cm}^{3}\right)$ were below the $107.34 \mathrm{~cm}^{3}$ threshold identified in the survey. The weights of the transmitters $(0.23 \mathrm{~kg})$ and the smartphones/transceivers $\left(0.17 \mathrm{~cm}^{3}\right)$ were also below the $1.16 \mathrm{~kg}$ threshold identified in the survey [5].

\subsection{Evaluation of the Framework with Existing Sensing Infrastructure}

\subsubsection{Localization Accuracy}

For situation 2, where there is existing sensing infrastructure at emergency scenes, the localization framework would implement the IMLE algorithm for location computation. In two field tests conducted for scenario 1 in situation 2, the IMLE algorithm achieved an average accuracy of $84.6 \%$ at the room level and $1.85 \mathrm{~m}$ at the coordinate level. In two field tests conducted for scenario 2 in situation 2, the IMLE algorithm achieved an average accuracy of $86.2 \%$ at the room level and $2.07 \mathrm{~m}$ at the coordinate level. The results are summarized in Table 2. The accuracies were consistent across the two scenarios that involved sensing areas of different sizes and shapes. Test results also showed that $62.5 \%$ of incorrect room-level estimations fell within neighboring rooms, suggesting that a secondary estimation of a target's locations could be possible if the initial estimation turned out to be incorrect. 
Table 2: Evaluation of the accuracy of the IMLE algorithm in the field tests

To further evaluate the performance of the IMLE, this algorithm is compared to two other localization algorithms. The first algorithm is the widely used maximum likelihood estimation (MLE) algorithm. It locates targets by MLE based triangulation, and does not take into account the impact of building geometries on signal propagation. The second algorithm integrates the widely used proximity based algorithm [40,41], assuming a target is located at its nearest neighboring transmitter, and offsets the impact of signal attenuations through walls with an iterative process. This algorithm is named as the iterative proximity (IP) algorithm in this paper. These two algorithms were selected for comparison as they share important characteristics (MLE based computation, an iterative process) with the IMLE algorithm, and at the meantime integrate existing indoor localization algorithms (i.e., MLE and proximity) that have been extensively investigated in the prior research [55].

The results, which are also summarized in Table 2, showed that the accuracies of the MLE and IP were significantly lower than those of the IMLE both at the room level and the coordinate level in both scenarios. The failure of the MLE was due to its negligence of RF signal attenuations caused by walls, and the failure of the IP was due to its heavy reliance on a high density of transmitters that was unavailable in the field tests. This comparison highlights the advantages of the IMLE algorithm, particularly its capability of offsetting the impact of walls and its insensitivity to the amount of devices when existing sensing infrastructure is accessible at building emergency scenes.

\subsubsection{Robustness against Partial Loss of Existing Transmitters}

The test data were further analyzed to evaluate the robustness of the IMLE algorithm against occurrence of losses of devices, including transmitters and transceivers. Beginning with the initial deployment, the analysis process was the same as the process for analyzing the robustness of the EASBL. A minimum of 3 transmitters and 3 transceivers were needed for the framework to implement the IMLE algorithm. This subsection discusses the findings about the robustness against loss of transmitters. The results are shown in Figure 7. The findings about the robustness against loss of transceivers are discussed in the next subsection.

Figure 7: Robustness against partial loss of existing transmitters in the field test 
As can be seen in Figure 7, the IMLE algorithm was proven robust against partial loss of transmitters. In scenario 1 , the room-level accuracy remained above $80 \%$ when up to 3 transmitters, or $27.2 \%$ of all transmitters, were removed. Similarly, in scenario 2, the room-level accuracy remained above $80 \%$ when up to 4 transmitters, or $30.8 \%$ of all transmitters, were removed. The impact of loss of transmitters became dominant when only 4 or fewer transmitters were left. The results suggested that a satisfactory accuracy could be achieved with a small number of transmitters, and that extra transmitters added to the network, while not making significant contribution to the accuracy, could provide additional robustness to the localization solution.

\subsubsection{Robustness against Partial Loss of Existing Transceivers}

It was found in the field test that in both scenarios the room-level accuracy was not significantly impacted by the loss of 2 or fewer transceivers, with the accuracy remaining above $75 \%$ and $80 \%$ in scenario 1 and scenario 2, respectively. However, unlike the simulation, the field tests reported large reductions in the accuracy to an unreliable level of below 55\% when a third transceiver was removed. The impact of the loss of transceivers was highly consistent across the two scenarios, as shown in Figure 8.

Figure 8: Robustness against partial loss of existing transceivers in the field test

\subsubsection{Ease of On-Scene Deployment, Computational Speed, and the Size and Weight of Devices}

In addition to the provision of satisfactory accuracy and robustness, the IMLE was proven to be capable of satisfying the other three important requirements identified in the survey. First, the IMLE, unlike the EASBL, does not require computation of ad-hoc network deployment plans and the deployment of the networks. Therefore, it could be easily set up and implemented at emergency scenes. Second, the computational time for updating the location information did not took more than five seconds based on observations in the field tests. Since the field tests used the same prototype, it could satisfy the requirements regarding the size and weight of devices as discussed above. 


\section{Discussions}

This section discusses the assessment results reported from both simulations and field tests. It needs to be noted that, in order to better depict the performance of the framework and given the acceptable repeatability of simulations, the accuracies reported in simulations are presented as ranges instead of point values. They are confidence intervals of the average accuracy, calculated at a $95 \%$ confidence level.

\subsection{Comparison between Simulations and Field Tests}

\subsubsection{Localization Performance with No Existing Sensing Infrastructure}

The reported accuracies of the EASBL, in both simulations and field tests, under the situation where there is no existing sensing infrastructure, are summarized in Table 3. As can be seen in the table, both in the simulations and the field tests, the performance of the framework was consistent across the two scenarios, despite the variations in the size and layout of the sensing areas. The discrepancies of the room-level accuracy were less than $0.9 \%$, and the discrepancies of the coordinate-level accuracy were less than $0.21 \mathrm{~m}$. The accuracies reported in the field tests were slightly lower than those reported in the simulation. The difference could have been caused by multiple reasons, such as the existence of complex environmental factors that interfered with the propagation of RF signals, the influence of targets' movements, and variations in the test subjects' gestures of holding the smartphones. In addition, it was observed both in the simulations and field tests that for a large portion of the targets that were incorrectly estimated with the EASBL at the room level, their estimated rooms were neighboring their actual rooms $(83.6 \%$ and $41.7 \%$ in simulations and field tests, respectively).

Table 3: Localization accuracy under situation 1

Moreover, the measured robustness of the framework in simulations was generally consistent with the robustness measured in the field tests. The magnitude of reduction of the room-level accuracy with increased loss of deployed transmitters was comparable between simulations and field tests, as can be seen in Figure 9. Given the same number of remaining deployed transmitters, the average difference of the room-level accuracy between the simulation and the field tests was $1.8 \%$ with slight variations. The reduction of accuracy occurred at a lower rate in scenario 2 , due to the larger number of transmitters initially deployed in the sensing area. In general, it can be 
concluded that the overall performance of the EASBL was comparable between simulations and field tests.

Figure 9: Comparison of robustness between simulations and field test

\subsubsection{Localization Performance with Existing Sensing Infrastructure}

The reported accuracies of the IMLE, in both simulations and field tests, under the situation where there is existing sensing infrastructure, are summarized in Table 4. Both in simulations and field tests, the accuracy again was consistent across the two scenarios. The discrepancies of the room-level accuracy were less than $1.6 \%$, and the discrepancies of the coordinate-level accuracy were less than $0.22 \mathrm{~m}$. The accuracies were expectedly lower than those reported in simulations, by approximately $10 \%$ at the room level and $1.2 \mathrm{~m}$ at the coordinate level. These differences were the likely results of various impacts discussed in the previous subsection. In addition, it was observed both in the simulations and field tests that for a large portion of the targets that were incorrectly estimated at the room level, their estimated rooms were neighboring their actual rooms $(87.1 \%$ and $62.5 \%$ in the simulation and the field tests, respectively).

Table 4: Localization accuracy under situation 2

A comparison of the robustness observed in the field tests to that reported in the simulations, as illustrated in Figure 10, showed that the IMLE algorithm was more robust in the simulated environment. Given the same extent of loss of transmitters, the reduction in the room-level accuracy was more significant (7.1\% higher on average) in the real-world environment, especially when the loss exceeded half of the total transmitters (up to $18.1 \%$ higher). Consequently, while a minimum of 3 transmitters would suffice to ensure a room-level accuracy of above $70 \%$ in the simulation in both scenarios, a minimum of 5 and 6 transmitters were required in scenario 1 and scenario 2, respectively, to achieve the same level of accuracy in the field tests.

Figure 10: Comparison of robustness against loss of transmitters between simulations and field tests

As for the robustness against the loss of transceivers, it was found that there was a discrepancy between simulations and field tests regarding the impact of removing a third transceiver. This comparison is illustrated in Figure 11. The figure shows that the magnitude of the accuracy 
reduction was generally comparable between simulations (blue lines) and field tests (red lines) in both scenario 1 (circles) and scenario 2 (triangles), except when a third transceiver was removed.

Figure 11: Comparison of robustness against loss of transceivers between simulations and field tests

\subsection{Comparison between the Two Algorithms}

This subsection compares the performance of the two algorithms under two situations. It is important to emphasize that two algorithms are designed for the localization framework with different intentions for different situations. This inherently determines the different performances of the framework even when used in the same building fire emergency scenarios.

The accuracies of the framework under two situations, both in simulations and field tests, are summarized in Table 5. As can be seen in the table, the accuracy under situation 1, where the EASBL was implemented, was generally lower than the accuracy under situation 2, where the IMLE was implemented. In simulations, the room-level accuracy under situation 1 was $8.0 \%$ and 7.9\% lower than that under situation 2 in scenario 1 and scenario 2, respectively; the coordinatelevel accuracy under situation 1 was $1.06 \mathrm{~m}$ and $0.73 \mathrm{~m}$ lower than that under situation 2 in scenario 1 and scenario 2, respectively. In the field tests, the room-level accuracy under situation 1 was $1.8 \%$ and $2.5 \%$ lower than that under situation 2 in scenario 1 and scenario 2, respectively; the coordinate-level accuracy under situation 1 was $0.27 \mathrm{~m}$ and $0.22 \mathrm{~m}$ lower than that under situation 2 in scenario 1 and scenario 2 , respectively.

There are two reasons that explain the relatively lower accuracies under situation 1. First, the accuracy was one of the two objectives the EASBL aims to optimize. By selecting deployment plans that balance the accuracy and deployment effort, the EASBL did not demonstrate its full capacity in improving the accuracy. Second, the IMLE utilized more sensing infrastructure, including all transmitters in the same placement utilized by the EASBL, and a number of additional transceivers. The extended sensing infrastructure and the resulting richer sensor data contributed to the higher accuracy yielded by the IMLE. In addition, smaller discrepancies in the accuracy of the two algorithms were observed in the field tests than in the simulations. The cause of such reduced discrepancies is that the environmental factors that the simulation did not factor in, such as the existence of metal furniture and the movement of people, had more impact on the accuracy of the IMLE. Such stronger impact could result from the fact that the IMLE, unlike the 
EASBL, relied on a mapping between signal strength and physical distances, and the mapping was sensitive to the impact of the environmental factors.

Table 5: Comparison of the accuracy between the EASBL and the IMLE

The IMLE algorithm also showed better robustness. As can be seen in Figure 12 and Figure 13, in both scenario 1 (red solid lines) and scenario 2 (blue dashed lines), the EASBL (circles) experienced faster reductions in the room-level accuracy than the IMLE (triangles) when the number of deployed transmitters was gradually reduced. With the minimum number of deployed transmitters, i.e. 2 transmitters for the EASBL and 3 transmitters for the IMLE, the accuracy of the EASBL was below 20\%, while the accuracy of the IMLE was above $40 \%$. The higher robustness of the framework under situation 2 results from the use of the MLE method in the IMLE algorithm. The performance of the MLE method should not vary significantly with variances in the number of input data entries, if there are no outliers in the input data.

Figure 12: Comparison of the robustness of the two algorithms (simulation)

Figure 13: Comparison of the robustness of the two algorithms (field test)

The localization framework was proven to be deployable on scene within the $135 \mathrm{~s}$ threshold under both situations in the field tests. Under situation 1, the framework required extra time during the on-scene deployment to address the challenge of having to establish an ad-hoc sensor network. It is important to note that on one hand, the lack of the ability in the IMLE algorithm to address this challenge results in the IMLE algorithm not being applicable to situation 1; on the other hand, the EASBL algorithm is applicable to situation 2, but with reduced accuracy and robustness. The EASBL algorithm reduced the ad-hoc network deployment effort by $32.5 \%$ in scenario 1 and $31.7 \%$ in scenario 2 . In addition, the computational time for updating the location information was comparable across the two algorithms and in both cases far less than the $40.34 \mathrm{~s}$ threshold. The framework could use the same prototype under both situations in the field tests, and therefore always satisfied the thresholds of $107.34 \mathrm{~cm}^{3}$ and the $1.16 \mathrm{~kg}$ for device size and device weight, respectively. 


\section{Limitations}

While the localization framework has achieved promising results both in the simulation and the field tests, it bears a number of limitations that need to be noted. First, some caution is needed when generalizing the reported results. The framework has been tested for two scenarios in two situations and yielded consistent performances. However, it must be pointed out that the selected test bed building is not representative of all building types, nor are the two emergency scenarios representative of all emergency scenarios that could happen in the real world. Admittedly, the performance of the framework may differ when implemented in other buildings, especially those with drastically different construction types, interior spatial layouts, construction materials and furniture. For instance, room-level localization becomes more challenging in densely partitioned spaces filled with metal-made furniture than in open-plan spaces with few obstructions. The performance may also differ when the nature, scope and severity of the emergencies are different than those used in the simulation and the field tests. For instance, fire emergencies spreading across multiple floors are more likely to damage existing devices and leave less room for first responders to deploy ad-hoc networks, and would challenge the scalability of the localization framework. Further implementation of the framework in diverse test beds and emergency scenarios is necessary in order to perform more conclusive evaluation of the framework.

Furthermore, the framework was not tested at real building emergency scenes. Setting up a real emergency in the test bed building is prohibited by safety regulations. The framework could not be tested in fire departments' drills either due to liability and logistical issues. Such field tests, if could be carried out in the future, would provide valuable evidence to address the following seven critical questions regarding the performance of the algorithms when challenged by real emergencies: (1) How accurate and robust is the localization framework when challenged by hazards on emergency scenes? (2) What additional constraints would hazards on emergency scenes impose on the deployment of ad-hoc networks? (3) How reliable is the RF sensor data transmitted and received by devices functioning under high temperature? (4) To what extent are device losses likely to happen? (5) How reliable is the WiFi based or cellular network based communication between smartphones and the webserver? (6) How to quickly determine the sensing area boundary that is needed to initialize the localization framework? (7) How to best fit the devices into the coat or backpacks worn by first responders in order to protect the devices while avoiding intrusiveness? The answers to these questions would further clarify the validity and applicability of the framework. 
Equally important, the evaluation of the framework was based on a critical assumption that all targets would have access to mobile nodes that can collect RF signal data and transfer the data to a remote server. Admittedly, this assumption may not always be satisfied in real-world situations, especially for trapped occupants. However, this assumption would become more realistic if the framework is applied to certain types of buildings, such as government buildings and healthcare facilities, where employers can mandate the use of required mobile nodes. The prototype has proven that smartphones can be used as the mobile nodes, avoiding intrusiveness to the occupants. Implementing the framework among first responders would be easier, as first responders have indicated in the survey the feasibility of carrying mobile nodes that do not exceed the size and weight limits during their operations.

The framework could be integrated into the incident command system (ICS) concept. The ICS provides a systematic tool used for the command, control, and coordination of emergency response operations, and is widely endorsed, sometimes mandated, by the federal agencies in the U.S. that have emergency response responsibilities. The data flow and interface of the localization framework need to be redesigned to be interoperable with the ICS, so that the localization information can be shared by multiple agencies collaborating in emergency response operations.

In addition, as discussed in [50], the processing of geometries extracted from BIM models when implementing the EASBL was observed in the field tests to be prohibitively time-consuming. There are several potential solutions to address this limitation: one can test other BIM tools that can process building geometries faster, or extract geometries from building models and process them with a customized code, or simply wait for the vendor to improve the computational efficiency of the BIM tool. Lastly, both the IMLE algorithm and the EASBL algorithm rely on BIM as a source of building information. Currently the accessibility of first responders to BIM is still low, though they indicated a foreseeable increase of the accessibility in the interviews.

Finally, the framework is based on and applicable to RF technologies only. RF technologies, which include but are not limited to RFID, WLAN, UWB and WSN, are rapidly evolving. The framework is essentially designed for any type of RF technologies. Those running at frequencies compatible with onboard sensors in smartphones are preferred, so that the implementation of the framework requires building occupants to carry no additional devices and is hence non-intrusive. However, the framework is not intended to be used with competing localization technologies such as inertial navigation system (INS). The algorithms are designed to process RF signal data, and 
are not capable of processing other types of data e.g., inertial sensor data. Admittedly, there is possibility that, with their new development in the future, these competing technologies may outperform the RF technologies in indoor localization. It therefore requires future research to extend the capacities of the framework, by examining how the competing technologies can be integrated in the framework to reduce its technology-dependence and better satisfy the indoor localization requirements.

To summarize, the evaluation of the localization framework presented in this paper serves as a feasibility study that demonstrates the potential of using the framework to locate first responders and trapped occupants. With the advancement of technological tools, the framework can be further improved to overcome the above limitations and perform better.

\section{Conclusions}

This paper presents a continuous effort of previous research of the authors in developing an indoor localization framework to support building emergency response operations. The ultimate objective is to provide first responders with accurate, robust and real-time indoor location information so that first responders can prioritize spaces that are more likely to have occupants when planning the search and rescue routes, and increase their own safety during the operations and avoid getting lost in buildings as well as avoiding the associated fatalities and injuries. The framework uses RF technologies, and selects from two innovative localization algorithms to implement depending on the availability of existing sensing infrastructure at building emergency scenes.

This paper provides an assessment of the framework in field tests, and synthesizes results, collected from different building emergency scenarios and situations, both in simulations and field tests. In simulations, the framework yielded room-level accuracies of above $87.0 \%$ and coordinate-level accuracies of above $1.78 \mathrm{~m}$ under situation 1, and room-level accuracies of above $95.0 \%$ and coordinate-level accuracies of above $0.84 \mathrm{~m}$ under situation 2 . In the field tests, the framework yielded room-level accuracies of above $82.8 \%$ and coordinate-level accuracies of above $2.29 \mathrm{~m}$ under situation 1, and room-level accuracies of above $84.6 \%$ and coordinate-level accuracies of above $2.07 \mathrm{~m}$ under situation 2 . The ease of on-scene deployment of the framework is addressed by the design of the EASBL algorithm that takes reducing deployment effort as one of the algorithm's two objectives. The framework yielded promising robustness both in the simulation and the field tests, by ensuring satisfactory accuracies upon partial loss of the sensing network. Moreover, the framework has been proven capable of meeting the requirements on 
computational speed and the size and weight of devices in the field tests. The assessment demonstrates that this localization framework is promising to achieve the above objective, and hence improve the efficiency and safety of building emergency response operations.

There are several limitations, such as limited representativeness of the test bed and emergency scenarios, simplicity of real building emergency situations, requirement on the use of mobile nodes, and incompatibility with competing indoor localization technologies. These limitations will be addressed in future research, to further improve the performance of the framework and advance this line of research.

\section{Acknowledgments}

This material is based upon the work supported by the National Science Foundation under Grant No.1201198. Any opinions, findings, and conclusions or recommendations expressed in this material are those of the author(s) and do not necessarily reflect the views of the National Science Foundation.

\section{References}

[1] MJ. Karter. Fire Loss in the United States during 2013, National Fire Protection Association, Fire Analysis and Research Division, Quincy, MA (2014).

[2] USFA. Fire-Related Firefighter Injuries Reported to NFIRS, U.S. Fire Administration, Emmitsburg, Maryland (2011).

[3] RF Fahy. U.S. FIRE SERVICE FATALITIES IN STRUCTURE FIRES, 1977-2009, National Fire Protection Association, Quincy, MA (2010).

[4] USFA. Firefighter Fatalities in the United States in 2011, U.S. Fire Administration, Emmitsburg, Maryland (2012).

[5] N Li, Z Yang, A Ghahramani, B Becerik-Gerber, L Soibelman. Situational awareness for supporting building fire emergency response: Information needs, information sources, and implementation requirements, Fire Saf.J. 63 (2014) 17-28.

[6] S Voigt, T Kemper, T Riedlinger, R Kiefl, K Scholte, H Mehl. Satellite image analysis for disaster and crisis-management support, IEEE Trans. Geosci. Remote Sens. 45 (2007) 1520-1528.

[7] R Harig, G Matz, P Rusch, H Gerhard, J Gerhard, V Schlabs. Infrared remote sensing of hazardous vapours: Surveillance of public areas during the FIFA football world cup 2006, Sensors, 
and Command, Control, Communications, and Intelligence (C3I) Technologies for Homeland Security and Homeland Defense VI, (2007), April 9-12.

[8] R Harig, P Rusch, H Peters, J Gerhard, R Braun, S Sabbah, et al. Field-portable imaging remote sensing system for automatic identification and imaging of hazardous gases, Remote Sensing of Clouds and the Atmosphere XIV, (2009), August 31-September 1.

[9] Z Zhong, N Jing, Q Wu, Y Gao. Integration of GIS/RS/GPS for urban fire response, 2012 International Conference on Computer Vision in Remote Sensing, CVRS 2012, (2012), December 16-18.

[10] H Yao, W Dong, D Liang, A Rogner, J Lai. Application of GIS on emergency rescue, 5th Conference on Performance-Based Fire and Fire Protection Engineering, (2011), December 7-9.

[11] C Wu, L Chen. 3D spatial information for fire-fighting search and rescue route analysis within buildings, Fire Saf.J. 48 (2012) 21-29.

[12] G Kogut, G Ahuja, EB Pacis, D Carroll, J Giles, B Rogers, et al. Sensor fusion for automatic detection of human presence, 1st Joint Emergency Preparedness and Response/Robotic and Remote Systems Topical Meeting, (2006), February 11-16.

[13] S Kim, R Maclejewski, K Ostmo, EJ Delp, TF Collins, DS Ebert. Mobile analytics for emergency response and training, Information Visualization, 7 (2008) 77-88.

[14] P Mitra, C Poellabauer. Emergency response in smartphone-based mobile Ad-Hoc networks, 2012 IEEE International Conference on Communications, ICC 2012, (2012) June 10-15.

[15] A Lo, L Xia, I Niemegeers, T Bauge, M Russell, D Harmer. EUROPCOM - an ultra-wideband (UWB)-based ad hoc network for emergency applications, Proc. of VTC/Spring - 2008 IEEE 67th Vehicular Technology Conference, (2008), May 11-14, Singapore.

[16] H Akcan, C Evrendilek. GPS-free directional localization via dual wireless radios, Comput.Commun. 35 (2012) 1151-1163.

[17] J Rantakokko, J Rydell, P Stromback, P Handel, J Callmer, D Tornqvist, et al. Accurate and Reliable Soldier and First Responder Indoor Positioning: Multisensor Systems and Cooperative Localization, IEEE Wireless Communications. 18 (2011) 10-18.

[18] A Chandra-Sekaran, P Weisser, K Muller-Glaser, C Kunze. A comparison of Bayesian filter based approaches for patient localization during emergency response to crisis, 2009 Third International Conference on Sensor Technologies and Applications (SENSORCOMM), (2009), June 18-23.

[19] J Duckworth, D Cyganski, S Makarov, W Michalson, J Orr, V Amendolare, et al. WPI precision personnel locator system - Evaluation by first responders, 20th International Technical Meeting of the Satellite Division of The Institute of Navigation 2007 ION GNSS 2007, September 25 - 28. 
[20] A Cavanaugh, M Lowe, D Cyganski, RJ Duckworth. WPI precision personnel location system: Rapid deployment antenna system and sensor fusion for 3D precision location, Institute of Navigation - International Technical Meeting 2010, ITM 2010, January 25 - 27.

[21] JR Guerrieri, MH Francis, PF Wilson, T Kos, LE Miller, NP Bryner, et al. RFID-assisted indoor localization and communication for first responders, 2006 First European Conference on Antennas and Propagation Conference, (2008), Nov 6-10.

[22] U Ruppel, KM Stubbe, U Zwinger. Indoor Navigation Integration Platform for firefighting purposes, 2010 International Conference on Indoor Positioning and Indoor Navigation (IPIN 2010), (2010), Sep 15-17.

[23] F Peña-Mora, AY Chen, Z Aziz, L Soibelman, LY Liu, K El-Rayes, et al. Mobile Ad Hoc NetworkEnabled Collaboration Framework Supporting Civil Engineering Emergency Response Operations, J.Comput.Civ.Eng. 24 (2010) 302-312.

[24] Jalal Mapar. Tracking emergency responders in challenging environments. http://spie.org/x39740.xml?ArticleID=x39740, Last accessed 2012, April 06.

[25] Exit Technologies. http://www.exit-technologies.com/draeger.php, Last accessed 2012, April 6.

[26] R Zhang, LM Reindl. Inertial localization system using unscented Kalman filter for 3D positioning, 2011 4th International Congress on Image and Signal Processing (CISP 2011), (2011), Oct 15-17.

[27] K Yamanaka, M Kanbara, N Yokoya. Localization of walking or running user with wearable 3D position sensor, 17th International Conference on Artificial Reality and Telexistence, ICAT 2007, (2007), Nov 28-30.

[28] M Akula, S Dong, VR Kamat, L Ojeda, A Borrell, J Borenstein. Integration of infrastructure based positioning systems and inertial navigation for ubiquitous context-aware engineering applications, Advanced Engineering Informatics. 25 (2011) 640-655.

[29] F Dovis, R Lesca, D Margaria, G Boiero, G Ghinamo. An assisted high-sensitivity acquisition technique for GPS indoor positioning, 2008 IEEE/ION Position, Location and Navigation Symposium - PLANS 2008, (2008), May 05.

[30] Y Liu, S Tian. Research of indoor GPS signals acquisition algorithm, 2008 4th International Conference on Wireless Communications, Networking and Mobile Computing (WiCOM), (2008), Oct 12-14.

[31] K Ozsoy, A Bozkurt, I Tekin. 2D indoor positioning system using GPS signals, 2010 International Conference on Indoor Positioning and Indoor Navigation (IPIN 2010), (2010), Sep 15-17. 
[32] S Hijikata, K Terabayashi, K Umeda. A simple indoor self-localization system using infrared LEDs, 2009 Sixth International Conference on Networked Sensing Systems (INSS 2009), (2009), Jun 17-19.

[33] S Tao, M Kudo, H Nonaka, J Toyama. Recording the activities of daily living based on person localization using an infrared ceiling sensor network, 2011 IEEE International Conference on Granular Computing, GrC 2011, (2011) November 8-10.

[34] D Hauschildt, N Kirchhof. Advances in thermal infrared localization: challenges and solutions, 2010 International Conference on Indoor Positioning and Indoor Navigation (IPIN 2010), (2010), Sep 15-17.

[35] R Ye, S Redfield, H Liu. High-Precision Indoor UWB Localization: Technical Challenges and Method, 2010 IEEE International Conference on Ultra-Wideband (ICUWB 2010), (2010), Sep 2023.

[36] H Xiong, H Song, Z Lai, J Zhang, K Yi. A novel indoor localization scheme, 2010 12th IEEE International Conference on Communication Technology (ICCT 2010), (2010), Nov 11-14.

[37] P Meissner, D Arnitz, T Gigl, K Witrisal. Analysis of an indoor UWB channel for multipathaided localization, 2011 IEEE International Conference on Ultra-Wideband (ICUWB 2011), (2011), Sep 14-16.

[38] N Li, S Li, B Becerik-Gerber, G Calis. Deployment Strategies and Performance Evaluation of a Virtual-Tag-Enabled Indoor Location Sensing Approach, Journal of Computing in Civil Engineering. 26 (2012) 574-583.

[39] N Li, G Calis, B Becerik-Gerber. Measuring and monitoring occupancy with an RFID based system for demand-driven HVAC operations, Autom.Constr. 24 (2012) 89-99.

[40] X Luo, WJ O'Brien, CL Julien. Comparative evaluation of Received Signal-Strength Index (RSSI) based indoor localization techniques for construction jobsites, Advanced Engineering Informatics. 25 (2011) 355-363.

[41] A Pradhan, E Ergen, B Akinci. Technological assessment of radio frequency identification technology for indoor localization, J.Comput.Civ.Eng. 23 (2009) 230-238.

[42] Shih-Hau Fang, T Lin. Principal Component Localization in Indoor WLAN Environments, IEEE Transactions on Mobile Computing. 11 (2012) 100-10.

[43] A del Corte-Valiente, J Gomez-Pulido, O Gutierrez-Blanco. Efficient Techniques and Algorithms for Improving Indoor Localization Precision on WLAN Networks Applications, International Journal of Communications, Networks and System Sciences. 2 (2009) 645-51.

[44] S Taneja, A Akcamete, B Akinci, JH Garrett J., L Soibelman, EW East. Analysis of Three Indoor Localization Technologies for Supporting Operations and Maintenance Field Tasks, J.Comput.Civ.Eng. 26 (2012) 708-19. 
[45] HM Khoury, VR Kamat. Evaluation of position tracking technologies for user localization in indoor construction environments, Autom.Constr. 18 (2009) 444-57.

[46] K Yedavalli, B Krishnamachari. Sequence-based localization in wireless sensor networks, IEEE Transactions on Mobile Computing. 7 (2008) 81-94.

[47] R Priwgharm, P Chemtanomwong. A comparative study on indoor localization based on RSSI measurement in wireless sensor network, 2011 Eighth International Joint Conference on Computer Science and Software Engineering (JCSSE 2011), (2011), May 11-13.

[48] Jehn-Ruey Jiang, Chih-Ming Lin, Yi-Jia Hsu. Localization with Rotatable Directional Antennas for Wireless Sensor Networks, 2010 39th International Conference on Parallel Processing Workshops (ICPPW), (2010), Sep 13-16.

[49] K Yedavalli, B Krishnamachari. Sequence-based localization in wireless sensor networks, IEEE Transactions on Mobile Computing. 7 (2008) 81-94.

[50] N Li, B Becerik-Gerber, B Krishnamachari, L Soibelman. A BIM centered indoor localization algorithm to support building fire emergency response operations, Automation in Construction. 42 (2014) 78-89.

[51] N Li, B Becerik-Gerber, L Soibelman. Iterative Maximum Likelihood Estimation Algorithm: Leveraging Building Information and Sensing Infrastructure for Localization during Emergencies, Journal of Computing in Civil Engineering. Accepted (2014) DOI: 10.1061/(ASCE)CP.19435487.0000430 .

[52] Mikrotik. Routerboard RB951G-2HnD. http://routerboard.com/RB951G-2HnD. Last accessed 2014, Dec 29.

[53] Motorola. Droid X. www.motorola.com. Last accessed 2014, Dec 29.

[54] Autodesk. Revit overview. http://www.autodesk.com.hk/products/revit-family/overview. Last accessed 2014, Sep 26.

[55] N Li, B Becerik-Gerber. Performance-based evaluation of RFID-based indoor location sensing solutions for the built environment, Advanced Engineering Informatics. 25 (2011) 535546. 


\section{Table List:}

Table 1: Localization accuracy of the EASBL algorithm and the optimal placement with proximity schema in the field tests

Table 2: Evaluation of the accuracy of the IMLE algorithm in the field tests

Table 3: Localization accuracy under situation 1

Table 4: Localization accuracy under situation 2

Table 5: Comparison of the accuracy between the EASBL and the IMLE 
Table 1: Localization accuracy of the EASBL algorithm and the optimal placement with proximity schema in the field tests

\begin{tabular}{|c|c|c|}
\hline Performance Metrics & EASBL & $\begin{array}{l}\text { Optimal placement } \\
\text { with proximity schema }\end{array}$ \\
\hline \multicolumn{3}{|c|}{ Scenario 1} \\
\hline Room-level accuracy (\%) & 82.8 & 31.7 \\
\hline Coordinate-level accuracy (m) & 2.12 & 3.23 \\
\hline \multicolumn{3}{|c|}{ Scenario 2} \\
\hline Room-level accuracy (\%) & 83.7 & 39.3 \\
\hline Coordinate-level accuracy (m) & 2.29 & 3.06 \\
\hline
\end{tabular}


Table 2: Evaluation of the accuracy of the IMLE algorithm in the field tests

\begin{tabular}{|c|c|c|c|}
\hline \multirow[t]{2}{*}{ Performance Metrics } & \multicolumn{3}{|c|}{ Localization Algorithm } \\
\hline & IMLE & MLE & IP \\
\hline \multicolumn{4}{|c|}{ Scenario 1} \\
\hline Room-level accuracy (\%) & 84.6 & 51.7 & 27.8 \\
\hline Coordinate-level accuracy (m) & 1.85 & 3.98 & 7.49 \\
\hline \multicolumn{4}{|c|}{ Scenario 2} \\
\hline Room-level accuracy (\%) & 86.2 & 55.3 & 32.5 \\
\hline Coordinate-level accuracy (m) & 2.07 & 4.06 & 7.17 \\
\hline
\end{tabular}


Table 3: Localization accuracy under situation 1

\begin{tabular}{|c|c|c|c|c|}
\hline \multirow{2}{*}{ Scenarios } & \multicolumn{4}{|c|}{ Performance of the EASBL } \\
\cline { 2 - 5 } & \multicolumn{2}{|c|}{ Simulation } & \multicolumn{2}{c|}{ Field test } \\
\hline & $\begin{array}{c}\text { Room-level } \\
\text { accuracy (\%) }\end{array}$ & $\begin{array}{c}\text { Coordinate-level } \\
\text { accuracy (m) }\end{array}$ & $\begin{array}{c}\text { Room-level } \\
\text { accuracy (\%) }\end{array}$ & $\begin{array}{c}\text { Coordinate-level } \\
\text { accuracy (m) }\end{array}$ \\
\hline Scenario 1 & $87.0 \pm 3.6$ & $1.78 \pm 0.24$ & 82.8 & 2.12 \\
\hline Scenario 2 & $87.2 \pm 3.8$ & $1.57 \pm 0.22$ & 83.7 & 2.29 \\
\hline
\end{tabular}


Table 4: Localization accuracy under situation 2

\begin{tabular}{|c|c|c|c|c|}
\hline \multirow{2}{*}{ Scenarios } & \multicolumn{4}{|c|}{ Performance of the IMLE } \\
\cline { 2 - 5 } & \multicolumn{2}{|c|}{ Simulation } & \multicolumn{2}{c|}{ Field test } \\
\hline \multirow{2}{*}{} & $\begin{array}{c}\text { Room-level } \\
\text { accuracy (\%) }\end{array}$ & $\begin{array}{c}\text { Coordinate-level } \\
\text { accuracy (m) }\end{array}$ & $\begin{array}{c}\text { Room-level } \\
\text { accuracy (\%) }\end{array}$ & $\begin{array}{c}\text { Coordinate-level } \\
\text { accuracy (m) }\end{array}$ \\
\hline Scenario 1 & $95.0 \pm 2.5$ & $0.72 \pm 0.10$ & 84.6 & 1.85 \\
\hline Scenario 2 & $95.1 \pm 2.7$ & $0.84 \pm 0.11$ & 86.2 & 2.07 \\
\hline
\end{tabular}


Table 5: Comparison of the accuracy between the EASBL and the IMLE

\begin{tabular}{|l|c|c|c|c|}
\hline \multirow{2}{*}{} & \multicolumn{4}{|c|}{ Simulation } \\
\cline { 2 - 5 } & \multicolumn{2}{|c|}{ Scenario 1 (EASBL) } & \multicolumn{2}{c|}{ Scenario 2 (IMLE) } \\
\cline { 2 - 5 } & Situation 1 & Situation 2 & Situation 1 & Situation 2 \\
\hline \multirow{2}{*}{ Room-level accuracy $(\%)$} & $87.0 \pm 3.6$ & $95.0 \pm 2.5$ & $87.2 \pm 3.8$ & $95.1 \pm 2.7$ \\
\hline \multirow{2}{*}{ Coordinate-level accuracy (m) } & $1.78 \pm 0.24$ & $0.72 \pm 0.10$ & $1.57 \pm 0.22$ & $0.84 \pm 0.11$ \\
\hline \multirow{3}{*}{ Room-level accuracy $(\%)$} & \multicolumn{2}{|c|}{ Field test } & \\
\cline { 2 - 6 } & Scenario 1 (EASBL) & Scenario 2 (IMLE) \\
\cline { 2 - 6 } & Situation 1 & Situation 2 & Situation 1 & Situation 2 \\
\hline Coordinate-level accuracy (m) & 82.8 & 84.6 & 83.7 & 86.2 \\
\hline
\end{tabular}




\section{Figure List:}

Figure 1: Transmitter (a) and smartphone (b) used in the prototype

Figure 2: Interface of the localization application

Figure 3: Snapshot of the BIM platform

Figure 4: Data flow in the field tests

Figure 5: Simulation scenarios and device layouts

Figure 6: Assessment of robustness against partial loss of deployed transmitters

Figure 7: Robustness against partial loss of existing transmitters in the field test

Figure 8: Robustness against partial loss of existing transceivers in the field test

Figure 9: Comparison of robustness between simulations and field test

Figure 10: Comparison of robustness against loss of transmitters between simulations and field tests

Figure 11: Comparison of robustness against loss of transceivers between simulations and field tests

Figure 12: Comparison of the robustness of the two algorithms (simulation)

Figure 13: Comparison of the robustness of the two algorithms (field test) 


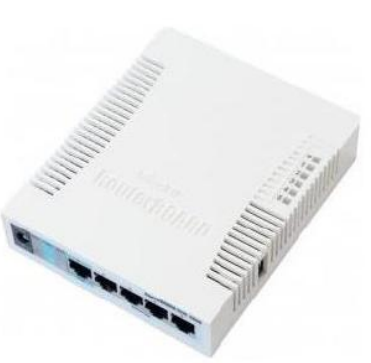

(a)

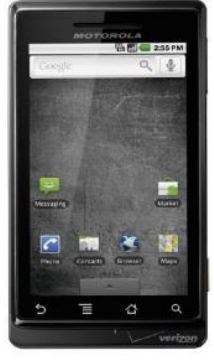

(b)

Figure 1: Transmitter (a) and smartphone (b) used in the prototype 


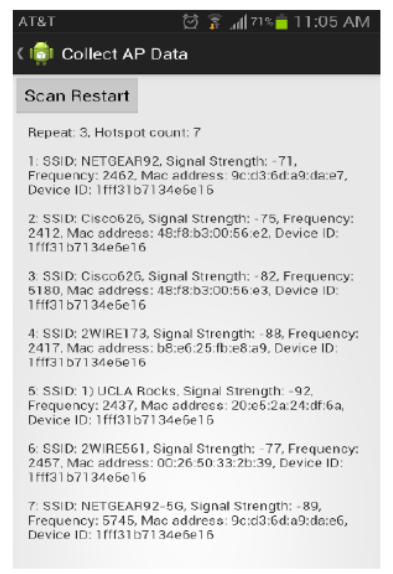

Figure 2: Interface of the localization application 


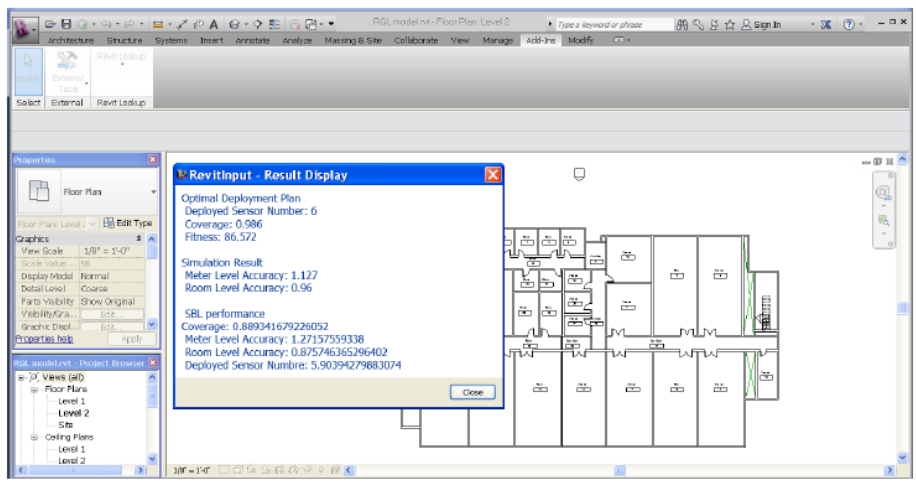

Figure 3: Snapshot of the BIM platform 


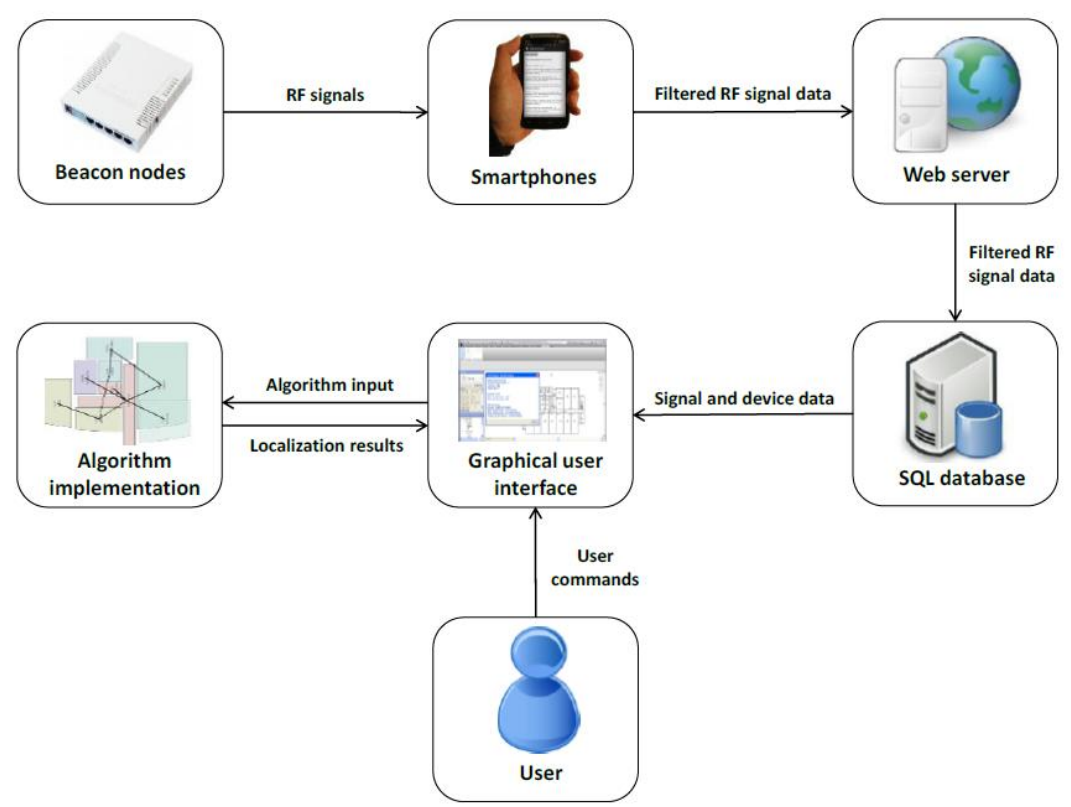

Figure 4: Data flow in the field tests 

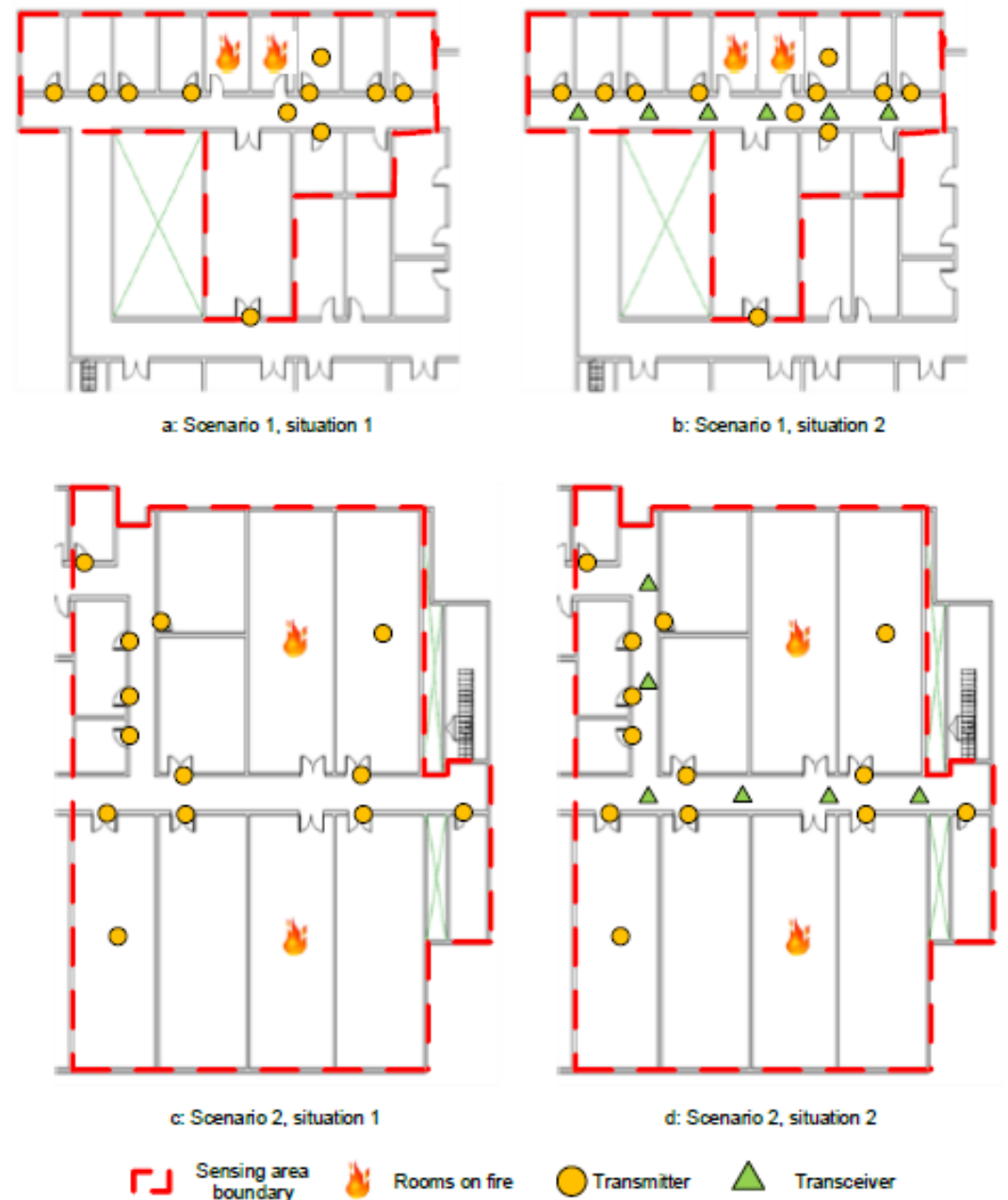

$\Gamma\rfloor \begin{gathered}\text { Sensing area } \\ \text { boundary }\end{gathered}$ Rooms on fire $\bigcirc$ Transmitter $\triangle$ Transceiver

Figure 5: Simulation scenarios and device layouts 


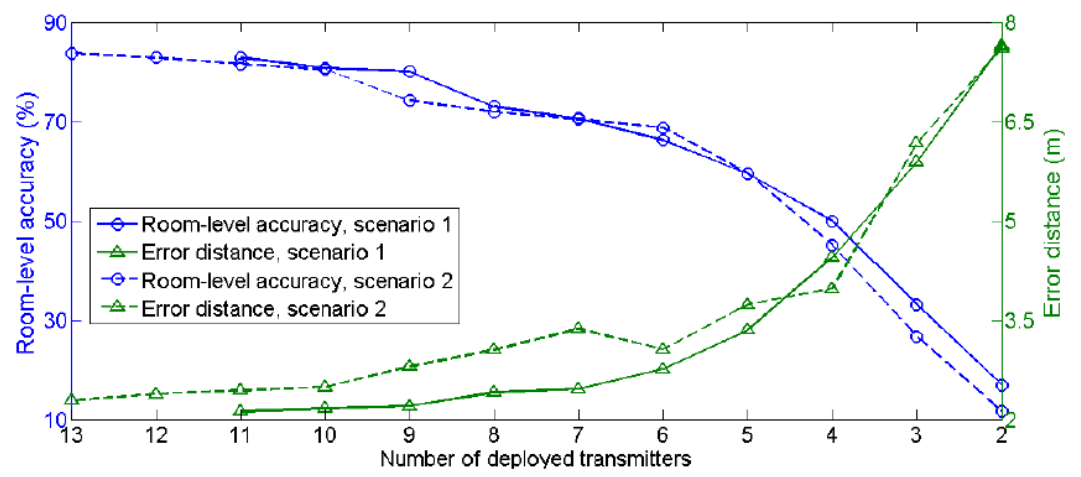

Figure 6: Assessment of robustness against partial loss of deployed transmitters 


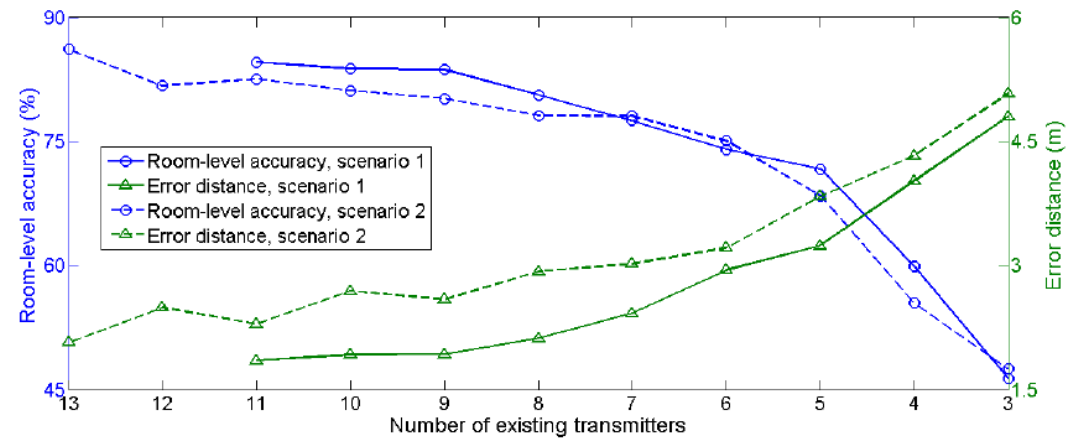

Figure 7: Robustness against partial loss of existing transmitters in the field test 


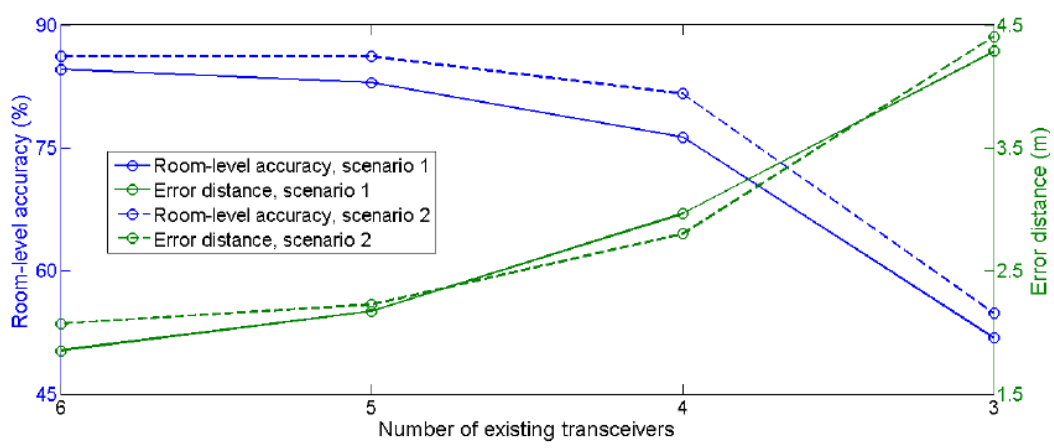

Figure 8: Robustness against partial loss of existing transceivers in the field test 


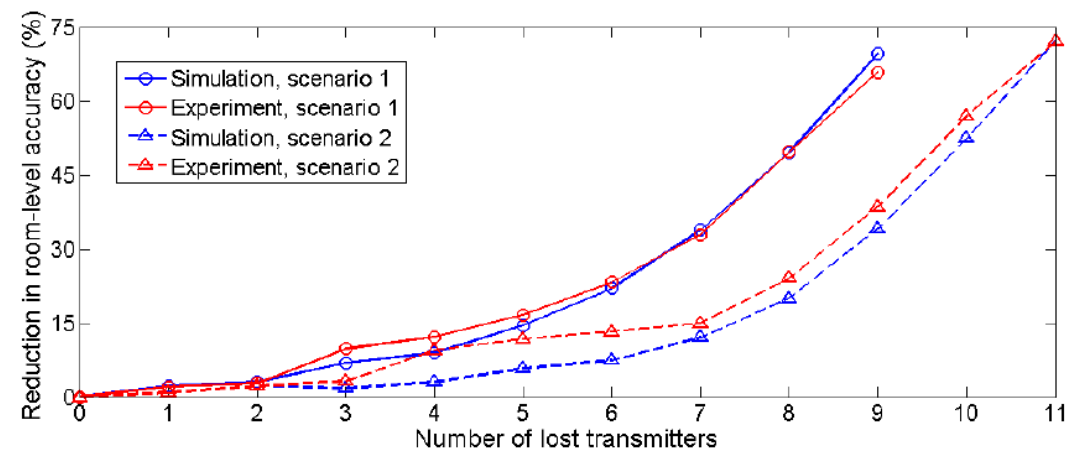

Figure 9: Comparison of robustness between simulations and field test 


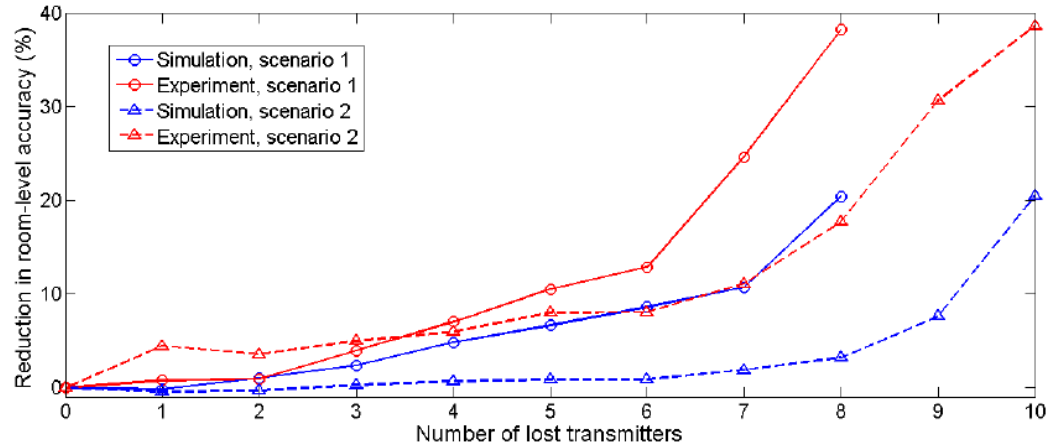

Figure 10: Comparison of robustness against loss of transmitters between simulations and field tests 


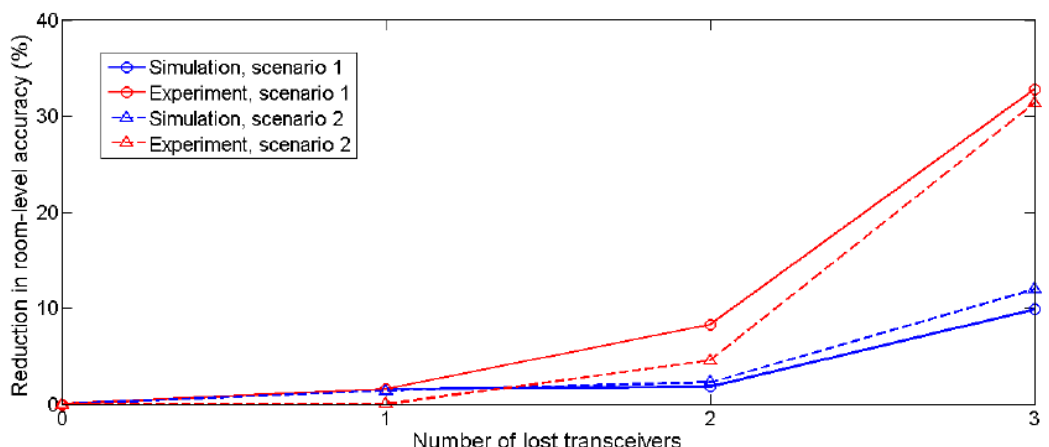

Figure 11: Comparison of robustness against loss of transceivers between simulations and field tests 


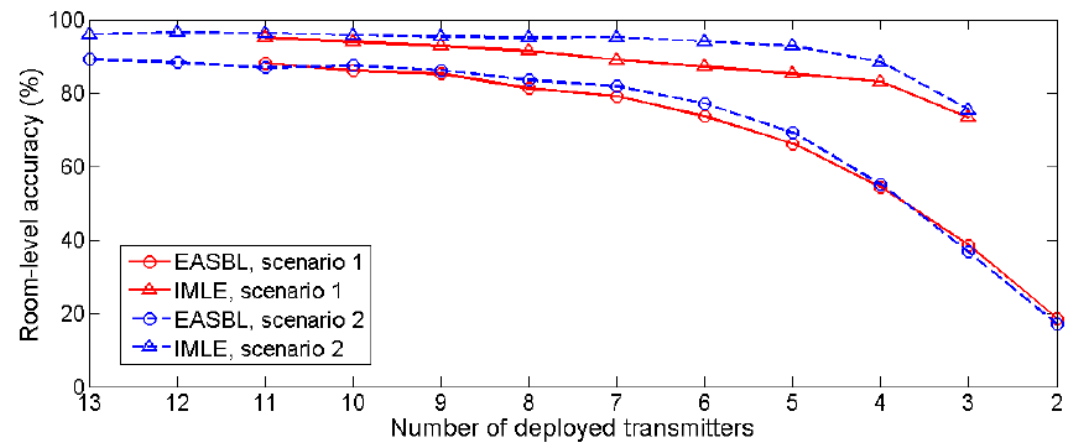

Figure 12: Comparison of the robustness of the two algorithms (simulation) 


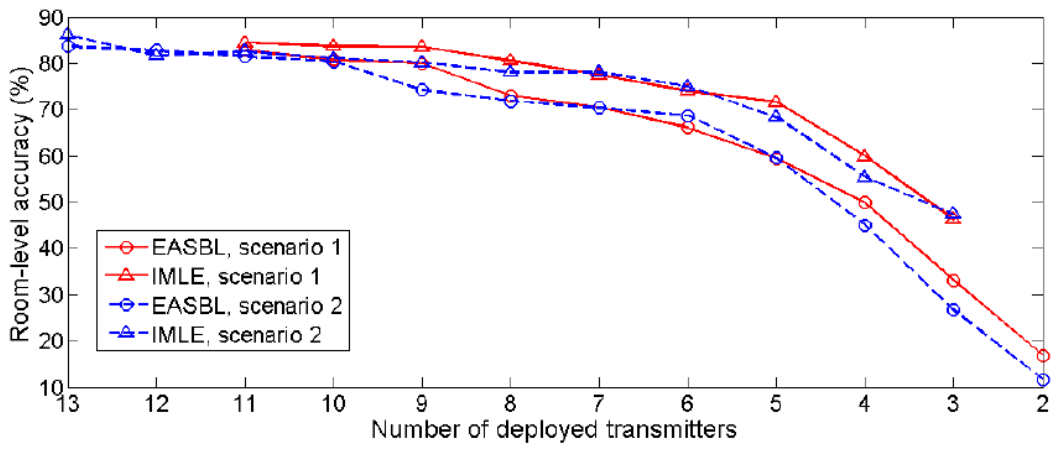

Figure 13: Comparison of the robustness of the two algorithms (field test) 